\title{
Experiencia en dos hospitales de tercer nivel de atención del suroccidente de Colombia en la aplicación del Registro Internacional de Trauma de la Sociedad Panamericana de Trauma
}

\author{
Experience of two first level hospitals in the southwest region of Colombia on \\ the implementation of the Panamerican Trauma Society International Trauma \\ Registry
}

\author{
Carlos A. Ordóñez MD'; Luis Fernando Pino MD²; Jorge W. Tejada MD³; Marisol Badiel MD; Jhon Harry Loaiza ${ }^{5}$; \\ lina V. Mata ; Michael B. Aboutanos, MD7
}

\section{R E S U M E N}

\begin{abstract}
Objetivo: Describir la experiencia en la implementación de un Sistema de Registro de Trauma (SRT) en dos hospitales en Cali, Colombia. Métodos: EI SRT incluye información prehospitalaria, hospitalaria y estatus de egreso del paciente. Cada hospital tiene una estrategia para la captura electrónica de datos. Se presenta un análisis descriptivo exploratorio durante un piloto de tres meses. Resultados: Se han registrado 3293 pacientes, 1626(49.4\%) del Hospital Público y 1613(50.6\%) en el Privado. $67.2 \%$ fueron hombres; edad promedio 30,5 20 años, 30,5\% menores de 18 años. Mortalidad global 3,52 \%. Causa más frecuente de consulta fueron las caídas (33,7\%); $11.6 \%$ fueron heridas por arma de fuego, la mortalidad en este grupo fue del 44.7\%. Conclusión: Se determinaron las necesidades para la implementación del SRT y los mecanismos para darle continuidad. El registro se convierte en una fuente de información para el desarrollo de la investigación. Se identificaron las causas de consulta, morbilidad y muerte por trauma que permitirá una mejor planeación de los servicios de urgencias y del sistema regional de trauma con el fin de optimizar y de reducir los costos de atención. A partir de este sistema de información de trauma se podrán plantear los ajustes indispensables para rediseñar el sistema de trauma y emergencias del suroccidente colombiano.
\end{abstract}

Descriptores: Heridas y traumatismos. Urgencias médicas. Base de datos. Sistema de registros. Registros electrónicos de salud.

\section{INTRODUCCIÓN}

E trauma es una epidemia global, en especial en países en vías de desarrollo. La región de América Latina tiene una incidencia de aproximadamente $11 \%$ del total de la mortalidad mundial asociada al trauma ${ }^{1}$. En la última década el trauma por causas externas y violentas representa la primera causa de muerte en la población menor de 44 años en Colombia. Cali tiene una tasa de mortalidad por lesiones personales de 81 por cien mil habitantes en el año $2010^{2}$, generando un grave problema de salud pública.

El conocimiento de la epidemiología del trauma es fundamental para identificar los factores asociados a la generación de lesiones de causa externa y crear las soluciones desde el punto de vista de la salud pública. Una de las herramientas disponibles para el monitoreo de las tendencias de este evento son los Sistemas de Registro de Trauma, utilizados primordialmente para identificar y evaluar la atención del trauma en todos sus niveles: hospitalario, local, regional y Estatal. En Estados Unidos, el National Trauma Data Bank es una institución con fundamento legal que lleva una estadística confiable y exacta de cualquier suceso relacionado con salud en dicho país ${ }^{3,4}$, de acceso fácil a la información con la finalidad de desarrollar estudios de investigación y análisis estadístico. Además, permite obtener en tiempo real estadísticas y mapas con la descripción de los múltiples eventos en salud, entre ellos el trauma. Mediante este sistema el estado Norteamericano monitorea los eventos y encamina políticas públicas en relación con los hallazgos ${ }^{4}$.

1. Profesor Asociado de Cirugía, Trauma y Cuidado Intensivo Universidad del Valle. Cirugía General, Trauma y Cuidado Intensivo Fundación Valle del Lili; 2. Cirujano General, Fellow en Cirugía de Trauma y Emergencias Universidad del Valle; 3. Médico Rural de la Unidad de Investigaciones Clínicas, Fundación Valle del Lili; 4. MSc, Directora Unidad de Investigaciones Clínicas, Fundación Valle del Lili; 5 . BSc Estadístico Unidad de Investigaciones Clínicas, Fundación Valle del Lili; 6. Fellow, International Trauma System Development Program, Department of Surgery, Division of Trauma, Critical Care and Emergency Surgery, Virginia Commonwealth University Medical Center, Virginia Commonwealth, USA; 7. MSc, Department of Surgery, Division of Trauma, CriticalCare and Emergency General Surgery, Virginia Commonwealth University Medical Center, Virginia Commonwealth, USA. 
A pesar del impacto del trauma en la morbilidad y la mortalidad en América Latina y Colombia, a la fecha no se cuenta con herramientas que de manera sistemática permitan observar y monitorear estos eventos. Los sistemas actuales de vigilancia epidemiológica son llevados como registros en papel, son poco confiables y no tienen continuidad. En el 2005 se planteó el piloto del Registro de Trauma de la Sociedad Panamericana de Trauma en Ecuador, sin embargo, esta experiencia no fue exitosa y fue descontinuada, debido a la falta de recursos ${ }^{5}$. Con este panorama, se planteó la segunda iniciativa latinoamericana en el marco del programa ITR/SPT-ITSDP en la ciudad de Cali, Colombia, a finales de noviembre de 2011. Este escenario responde a una necesidad de registrar de manera eficiente y confiable los eventos de trauma, dadas las condiciones de la ciudad y el interés de fortalecer el actual sistema de trauma regional². La experiencia se fortalece por la facilidad que ofrece un sistema de información electrónico, probado en diferentes países, y que indique las tendencias reales del trauma en la región para pensar en organizar un Sistema de Trauma en el sur-occidente colombiano. El objetivo es describir la experiencia en la implementación de un Sistema de Registro de Trauma (SRT) en dos hospitales en Cali, Colombia.

\section{MÉTODOS}

En octubre de 2011 se establece un convenio colaborativo entre ITR/SPT. El convenio incluye el desplazamiento a Colombia del Virginia Commonwealth University (VCU) para entrenar al personal que captura la información, coordinadores regionales del registro, estudiantes de medicina encargados y el líder clínico de cada institución. Una vez realizado esta capacitación, el ITR/SPT-ITSDP se compromete con monitorizar a la captura de datos, brindar soporte técnico a la plataforma y dar solución a problemas mediante teleconferencias. La captura de información se realizó en dos fases: del 21 de noviembre de 2011 al 4 de enero de 2012 en una plataforma de prueba y la definitiva a partir del 5 de enero de 2012.

Plataforma del programa de Registro Internacional de Trauma El Registro Internacional de Trauma es una base de datos electrónica basada en web, perteneciente a la Sociedad Panamericana de Trauma mediante el Programa Internacional de Sistemas de Trauma (ITR/SPT- ITSDP); es el componente internacional de la División de Trauma y Cuidado Crítico de la Virginia Commonwealth University (VCU) ${ }^{6}$. El software del SRT es una herramienta digital desarrollada para funcionar en red a través de internet, consta de un total de 244 variables distribuidas en 11 ventanas que agrupan los datos en módulos operativos como se menciona a continuación: Información demográfica; motivo de consulta; antecedentes y lugar del evento; mecanismos de lesión; información prehospitalaria; examen físico; imágenes; laboratorio; diagnóstico y manejo en urgencias; hospitalización; referencia y contra-referencia. De esta forma permite registrar el seguimiento diario del paciente hospitalizado, especificar si fue o no sometido a cirugía u otros procedimientos, calcular automáticamente ciertas variables como edad de acuerdo a la fecha de nacimiento, escalas e índices como la Escala de Coma de Glasgow, el Índice de Trauma Revisado (RTS), el Índice de Severidad de las Lesiones (ISS) y el estatus al egreso de cada paciente. Permite generar reportes estandarizados y otros específicos según la necesidad de información. Todos los diagnósticos clínicos y los mecanismos de lesión están codificados según la Clasificación Internacional de Enfermedades (CIE-10 ó ICD10 por sus siglas en inglés ${ }^{7,10}$.

\section{Sitios}

El Hospital Público (Hospital 1) es una entidad del estado, clasificado como centro de III nivel de atención y es el hospital de referencia de la red pública. Cuenta con un total de 750 camas, 143 designadas para la atención de los pacientes traumatizados que llegan a la institución y 38 camas de Cuidado Intensivo. Se estima que el número de ingresos totales al año en el servicio de urgencias por trauma es de 8.450 pacientes. En esta institución, el paciente durante el manejo de su hospitalización puede estar hospitalizado en 13 sitios diferentes entre salas de unidad de trauma, urgencias, observación, hospitalización general, cuidado intensivo, entre otros. Para el Sistema de Registro de Trauma (SRT) se estableció la estrategia de un coordinador general clínico (Fellow de trauma), 5 personas para la captura de información con perfil de tecnólogos en atención prehospitalaria de tiempo completo y que se distribuyen en turnos de 12 horas todos los días, incluyendo fines de semana y días festivos. La captura de la información es realizada desde urgencias y una vez el paciente es trasladado las diferentes salas del hospital; los datos son adquiridos de la historia clínica de papel. La información para el SRT es capturada en equipos portátiles dotados de conexión a red inalámbrica.

El Hospital Privado (Hospital 2) es una entidad privada, sin ánimo de lucro, que consta de 448 camas en general, de las cuales 120 son de Unidades de Cuidados Intensivos (UCI), 10 de ellas de manera casi exclusiva a trauma. Anualmente se realizan cerca de 10000 consultas por trauma en esta institución. Está equipado también con una Unidad de Reanimación en Urgencias adultos y pediátricas. La historia clínica es electrónica. Para el SRT la estrategia incluye al coordinador general clínico (Fellow de Cirugía de Trauma), un médico general en Servicio Social Obligatorio y un tecnólogo en atención prehospitalaria, los dos últimos de tiempo completo. Dos estudiantes de medicina asisten al personal ya descrito en tiempo parcial. La información de los ingresos por trauma, cirugías, 
hospitalización y egreso se captura directamente desde el sistema de historia clínica electrónica (SAP NetWeaver Business Client 1.0 SAP $\left.{ }^{\circledR}\right)$. La información para el SRT es capturada tanto en equipos portátiles dotados de conexión a red inalámbrica como en equipos de escritorio conectados a la red. Ambos hospitales son de carácter universitario. E estudio fue aprobado por el Comité de Ética de las dos instituciones.

\section{Análisis de Datos}

Para el análisis estadístico se exportó la base de datos en un archivo binario, con previa autorización del centro coordinador en Virginia. Este archivo es transferido a la plataforma de análisis en el software estadístico STATATM 10 (StataCorp, Texas-USA). Se realizó un análisis exploratorio descriptivo; las variables categóricas son presentadas en proporciones y las comparaciones entre ellas se realizarán con chi cuadrado o prueba exacta de Fisher según el caso. Las variables continuas se expresan como media, mediana, promedios, \pm desviación estándar y rangos intercuartílicos y serán analizadas con Student ìs T test ó Mann-Whitney test, según se cumplan o no los supuestos de normalidad.

\section{RESULTADOS}

Entre noviembre 21 de 2011 a febrero 18 de 2012, en los dos hospitales se registraron 3239 pacientes; 1626 (50.2\%) en el Hospital Público y 1613 (49.8\%) en el Hospital Privado, esto es, $36 \pm 13$ sujetos por día. La edad promedio global fue $30.5 \pm 20.3$ años; el $68.3 \%$ de los atendidos fueron hombres. Las principales causas de consulta fueron caídas (33.7\%), accidentes de tránsito (20.7\%) y lesiones personales de causa externa (19.8\%) (Figura 4). Se registraron 114 muertes (3.5\%). La tabla 1 muestra las características generales de la población registrada. La recolección muestra que en el mes de Enero2012 se capturó el 37.6\% de la información. Los días de la semana con mayor de número de pacientes ingresados

Tabla 1 - Características generales de los sujetos incluidos en el registro.

\begin{tabular}{|c|c|c|}
\hline Variable & $\mathrm{Ge}$ & neral \\
\hline Hombres, n (\%) & 2212 & $(69,5)$ \\
\hline Edad (años), (Promedio $\pm \mathrm{DE}$ ) & $30,5=$ & $\pm 20,3$ \\
\hline >18 años & 2251 & $(69,5)$ \\
\hline Rangos de edad, años, n (\%), ) & & \\
\hline $0-14$ & 709 & $(21,8)$ \\
\hline $15-24$ & 747 & $(23,1)$ \\
\hline $25-34$ & 645 & $(19,9)$ \\
\hline $35-44$ & 382 & $(11,8)$ \\
\hline $45-54$ & 269 & $(8,3)$ \\
\hline $55-64$ & 172 & $(5,3)$ \\
\hline $65-74$ & 91 & $(2,8)$ \\
\hline $75+$ & 224 & $(6,9)$ \\
\hline Causas de trauma & & \\
\hline Caídas & 1091 & $(33,7)$ \\
\hline Accidente de tránsito & 671 & $(20,7)$ \\
\hline Arma de fuego & 377 & $(11,6)$ \\
\hline Arma cortopunzante & 267 & $(8,2)$ \\
\hline Envenenamiento & 49 & $(1,5)$ \\
\hline Quemadura & 126 & $(3,9)$ \\
\hline Otro mecanismo & 544 & $(16,8)$ \\
\hline Días estancia Hospitalaria, Mediana (RIQ) & 4,5 & $(1-10)$ \\
\hline Días estancia en UCI, Mediana (RIQ) & 3,5 & $(1-8)$ \\
\hline Total de muertes, n (\%) & 114 & $(3,5)$ \\
\hline Causa de muerte, $\mathrm{n}(\%)$ & & \\
\hline Accidente de tránsito & 24 & $(21,1)$ \\
\hline Caída & 23 & $(23,2)$ \\
\hline Arma cortopunzante & 6 & $(5,2)$ \\
\hline Arma fuego & 51 & $(44,7)$ \\
\hline Otro mecanismo & 10 & $(8,7)$ \\
\hline Total de sujetos & 3239 & \\
\hline
\end{tabular}


fueron el domingo (17\%), el sábado con 477 (14.7\%) y el miércoles con 465 (14.3\%), figuras 1-3.

\section{hospital}

\section{Características sociodemográficas por}

En el Hospital Público comparado con el hospital privado el promedio de edad fue mayor $(31,8 \pm 21,8$ vs $29,1 \pm 19,2, p=0,006)$, hubo mayor proporción de hombres (73.5\% vs $62.9 \%, p<0.001)$, tabla 2 .

En general, comparado con el hospital privado, al hospital público ingresaron pacientes más severamente comprometidos según evaluación con el Índice de Severidad de Lesiones ISS (mediana 9 (rango intercuartílico 5-13) vs 3 $(1-6), p<0.001)$ y con la proporción de sujetos con ISS $>15$ ( $8.5 \%$ vs $5.8 \%, p=0.002$ ), mientras que hubo un tendencia a atender más pacientes críticos con ISS $>25$ en el hospital privado (3.2\% vs $2.1 \%, p=0.059)$, tabla 2 .

Los pacientes con ISS más altos corresponden a heridas por arma de fuego (11.6\%); por arma cortopunzante y cortocontundente (8.2\%). El trauma clasificado como otros incluyó pacientes con agresiones, contusiones y explosivos. La estancia hospitalaria total fue más prolongada en el hospital público comparado con el privado (5.5 (1-9) días vs 5 (1-11), p <0.001), pero la estancia es unidad de cuidado intensivo fue similar en ambos sitios ( $p=0.18)$, tabla 2 .

\section{Mortalidad}

La mortalidad global fue de $3.52 \%(n=114)$, siendo mayor en el Hospital Público comparada con el Privado ( $4.6 \%$ vs $2.4 \%, p<0,001)$. La principal causa de mortalidad en ambas instituciones fueron las lesiones personales por causa externa $499,9 \%$ distribuido así: heridas por arma de fuego $44,7 \%$ y arma cortopunzante $5,3 \%$ respectivamente), seguido por accidentes de tránsito $(21,1 \%)$, y caídas $(23,2 \%)$. Estratificado por severidad con ISS >15, la tasa de mortalidad en los dos hospitales fue similar, en el Público $29.7 \%$ y en el privado

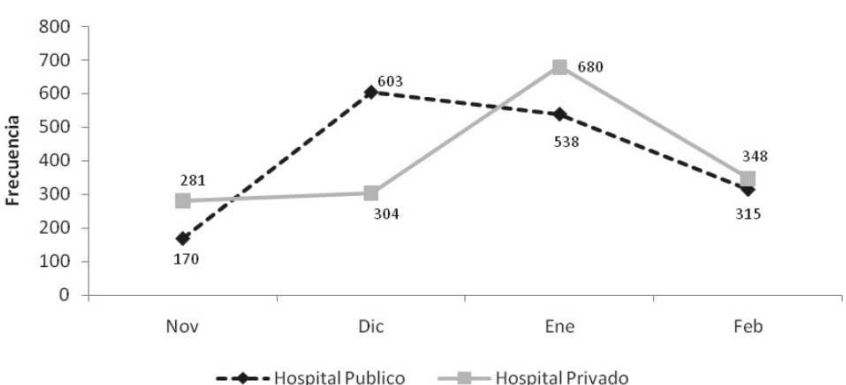

Figura 1 - Volumen de ingreso de pacientes registrados por mes de operación del registro de trauma.

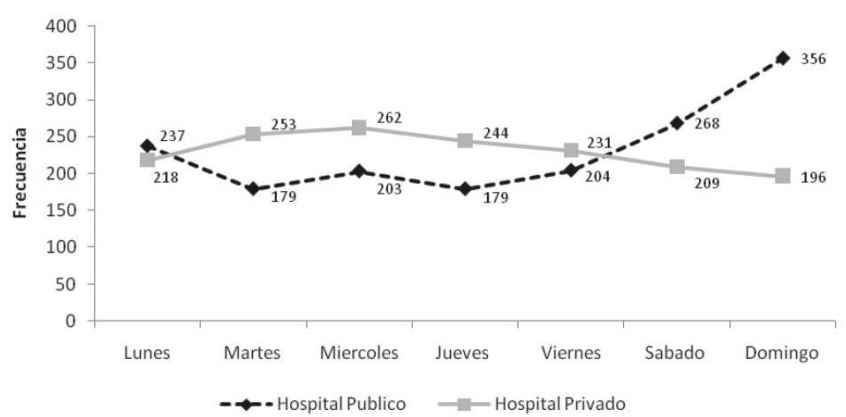

Figura 2 - Volumen de ingreso de pacientes registrados por día de la semana de operación del registro de trauma.

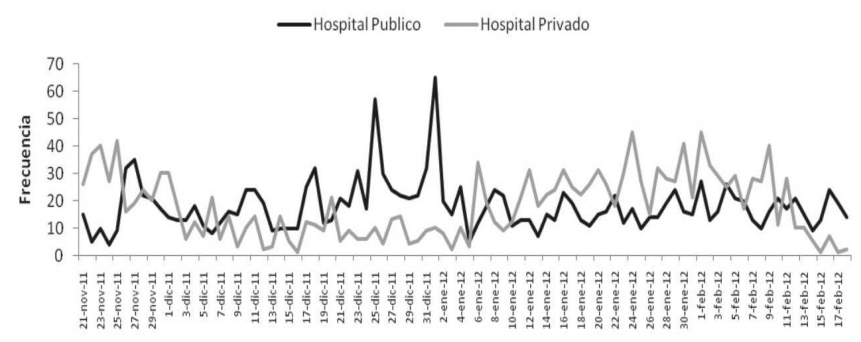

Figura 3 - Volumen de ingreso de pacientes registrados durante cada uno de los 90 días de operación del registro de trauma.

Tabla 2 - Características de los sujetos registrados por institución.

\begin{tabular}{|c|c|c|c|c|c|}
\hline \multirow[t]{2}{*}{ Variable } & \multicolumn{4}{|c|}{ Institución } & \multirow[b]{2}{*}{ Valor $\mathrm{p}$} \\
\hline & \multicolumn{2}{|c|}{ HOSPITAL PUBLICO(N = 1626) } & \multicolumn{2}{|c|}{ HOSPITAL PRIVADO $(\mathrm{N}=1613)$} & \\
\hline Hombres, n (\%) & 1196 & $(73,5 \%)$ & 1016 & $(62,9 \%)$ & $<0,001$ \\
\hline Edad, años, promedio $\pm D E$ & $31,8 \pm$ & $\pm \quad 21,8$ & $29,1 \pm$ & 19,2 & 0,006 \\
\hline e"18 años, n (\%) & 1145 & $(70,4)$ & 1107 & $(68,6)$ & 0,26 \\
\hline ISS, Mediana (RIQ) & 9 & $(4-13)$ & 3 & $(1-6)$ & $<0,001$ \\
\hline $\mid S S>15, n(\%)$ & 138 & (8.5) & 93 & $(5.8)$ & 0,002 \\
\hline $\mid S S>25, n(\%)$ & 35 & & $(2,1)$ & $52(3,2)$ & 0,059 \\
\hline Estancia Hospitalaria, días, Mediana (RIQ) & 5.5 & $(1-9)$ & 5 & $(1-11)$ & $<0,001$ \\
\hline Estancia en UCI, Mediana (RIQ) & 3,5 & $(2,5-6,5)$ & 4 & $(1-9)$ & 0,18 \\
\hline Muertes, n (\%) & 75 & $(4,62)$ & 39 & $(2,4)$ & $<0,001$ \\
\hline $\mid S S>15, n(\%)$ & $41 / 138$ & $(29,7)$ & $34 / 93$ & $(36,55)$ & 0,27 \\
\hline $\mid S S>25, n(\%)$ & $27 / 35$ & $(77,1)$ & $28 / 52$ & $(53,8)$ & 0,027 \\
\hline
\end{tabular}

Severidad y mecanismos del trauma. 
de $36,5 \%(p=0,27)$; mientras que para los pacientes más críticos definidos con ISS>25, la mortalidad del Hospital Público fue mayor que en el privado $(77,1 \%$ vs $53,8 \%$, $p=0,027)$.

\section{DISCUSIÓN}

La implementación en Cali de una plataforma electrónica en su fase de Pre-piloto y Piloto del Registro fue exitosa. Mediante dos estrategias diferentes y en dos tipos de hospitales se logró disponer de un sistema de información en tiempo real y con utilización oportuna de los registros.

\section{Historia y actualidad de los Registros Electrónicos de Trauma}

El primer Registro de Trauma Electrónico se desarrolló en el año de 1969 en el Hospital Cook Country de Chicago ${ }^{11}$ el cual fue la base para el Illinois Trauma Registry en el año de 1971; posteriormente, en el año de 1989, se institucionaliza el National Trauma Data Bank iniciado por el American College of Surgeons, actualmente con más de un millón de entradas en su base de datos y en colaboración con el CDC siendo extendido a toda la nación Norteamericana $4,11,12$. Si bien es cierto que este mismo se encuentra actualmente funcionando, para consolidar un Sistema Nacional de Registro de Trauma en Estados Unidos se necesitó de veinte años, con todas las dificultades que se hayan tenido en su momento. Varios países, además de Estados Unidos, llevan sus propios registros de trauma, como sucede en Canadá, Australia, Israel, Italia, Alemania, Japón, Holanda y Reino Unido ${ }^{13-15}$. Todos tienen establecido un protocolo de clasificación y adquisición de datos desde la atención Pre-hospitalaria, capacitación del personal encargado del registro, dotación de los equipos necesarios, financiación del sistema, desarrollo de un software adaptado a las necesidades de la región y un vínculo claro entre las actividades registradas y los objetivos de mejora en el sistema ${ }^{14}$

Sin embargo en los países en vía de desarrollo, son escasas las experiencias en registros de trauma en tiempo real a partir de plataformas electrónicas. Las experiencias documentadas parten de registros retrospectivos, depósitos de datos de cadáveres o encuestas poblacionales ${ }^{16,17}$. En Pakistán se presentó una iniciativa con la finalidad de llevar un registro a partir de los datos obtenidos en la Sala de Emergencias y al igual que en algunos países del continente africano se intentó desarrollar un SRT con el apoyo de la Agencia de Estados Unidos para el Desarrollo Internacional (USAID por sus cifras en inglés) pero ninguno se ha podido concretar con solidez ${ }^{14}$. En los países de América Latina se tiene reporte de un intento en Ecuador de aplicar el Registro Internacional de Trauma en el 2005, proyecto que se finalizó por finalización de recursos 5 .

\section{Iniciativas en Colombia}

La iniciativa entre la SPT y el VCU que incluyó dos hospitales en la ciudad de Cali es la primera en su género. Las experiencias en Colombia son similares a las identificadas en los países en desarrollo, en donde se tiene como referencia la presentación de reportes de tipo retrospectivo, con revisión de historias clínicas según un tipo específico de pacientes. Por su parte, el Sistema de Vigilancia Epidemiológica del Instituto Nacional de Salud vigila alrededor de 114 eventos de notificación obligatoria que incluye solamente enfermedades infecciosas y crónicas no transmisibles. Esta es la primera experiencia en Colombia que combina varias de las características fundamentales de un registro en salud en tiempo real y basado en la web, dando agilidad y credibilidad a los datos ${ }^{18}$.

Se destaca que no surge como una iniciativa del gobierno local o nacional, sino que se da como una propuesta académica de la Universidad del Valle y de las instituciones de salud que atienden buena parte de de los eventos de trauma que suceden en Cali. La experiencia y los excelentes resultados clínicos en el manejo del trauma son reconocidos a nivel nacional e internacional ${ }^{19,20}$.

\section{Características de las Instituciones de Salud}

Los resultados preliminares observados en cada institución reflejan la cobertura de cada una de ellas en cuanto al tipo de pacientes que ingresa y como está clasificada en la red de atención de la ciudad. Las instituciones de salud del Estado (o públicas) manejan un volumen importante de pacientes, que en su mayoría pertenecen a sistemas de afiliación subsidiada por el Estado (SISBEN 1 y 2) o que no están afiliados a ninguna Entidad Promotora de Salud (denominados también vinculados) que viven en zonas con altos índices de desocupación, baja escolaridad, pobreza y pandillas juveniles.

En los Hospitales Privados la atención se caracteriza por pacientes pertenecientes al régimen contributivo y medicina prepagada, grupo diferente al manejado en las Instituciones Públicas. Asimismo, cuentan en su mayoría con la ventaja de la aplicación del sistema informatizado de organización de la atención, brindando mayor disponibilidad de la información. Adicionalmente existe un convenio con el Ejército colombiano para la atención de los militares heridos en combate donde este hospital asume todos los costos.

\section{Utilidad de la Información Recopilada}

La principal causa de consulta son las caídas y trauma menor resultado de los accidentes laborales y escolares. La mortalidad por trauma menor es similar a otras poblaciones como en Australia y Estados Unidos 15,21,22. Siendo la ciudad de Cali una de las ciudades con más alta incidencia de violencia, agresiones y lesiones 
personales secundarias, se hubiese esperado que esta fuera la primera causa de consulta en ambos hospitales, sin embargo las lesiones de causa externa secundarias a heridas por arma de fuego y por armas cortopunzantes son la tercera causa de consulta después de las lesiones por accidente de tránsito que incluye lesiones a peatones y motociclistas principalmente. Estas tres causas principales se distribuyen de manera similar en ambas instituciones, siendo las heridas por arma de fuego la segunda causa en el hospital público (36\%) y la tercera en el privado (13.1\%).

Si bien las heridas por arma de fuego no fueron la principal causa de consulta, si generan la mayor mortalidad en la población. La mitad de las muertes registradas en el SRT: $44,7 \%$ fueron secundarias a heridas por arma de fuego y 5,2\% a arma cortopunzante, (Tabla 4). La segunda causa de muerte fueron los accidentes de tránsito, seguido por las caídas y otros mecanismos (contusiones, agresiones, envenenamiento, entre otros). La mortalidad general fue mayor en el Hospital público $(4,62 \%)$, donde también se registraron pacientes más severamente comprometidos: ISS $>15$ ( $8.5 \%$ vs $5.8 \%, p=0.002)$ y mayor porcentaje de pacientes heridos por arma de fuego ( $36 \%$ vs $13 \%$ ). La mortalidad en pacientes con ISS $>25$ fue menor en el hospital privado ( $53.8 \%$ vs $77.1 \%, p=0.027$ ). El promedio de estancia hospitalaria y en $\mathrm{UCl}$ en ambas instituciones es similar (tablas 1 y 2 ).

\section{Otros beneficios}

En esta primera fase de noventa días de aplicación del ITR/SPT-ITSDP se encontraron ventajas importantes como la confiabilidad y organización de los datos indexados, tomando como documento fuente la historia clínica; así como la disponibilidad inmediata de los mismos con la seguridad del acceso a la plataforma. La facilidad de generación de reportes mediante el generador de reportes, así como la facilidad de acceder al sistema desde cualquier punto de conexión a internet. Del mismo modo, el proyecto permitió comparar la versatilidad en la captura de información en un sistema de historia clínica digitalizada versus uno con historia clínica no digitalizada. Se encontró que en la institución con historia clínica digital se tiene mayor facilidad en la búsqueda de datos, menor consumo de tiempo y disminución de procesos para acceso a historias clínicas.

Con el registro de trauma se da inicio a un proceso de mejoramiento de la atención de los pacientes traumatizados. Teniendo en cuenta la versatilidad del Registro Internacional de Trauma, los beneficios que se pueden extraer del mismo, la disponibilidad y organización en datos justifica el costo de funcionamiento, es una inversión viable y necesaria a mediano y largo plazo, con importantes repercusiones en el ámbito médico, social y económico de las instituciones.

\section{Limitaciones}

Desde el punto de vista operativo, en cada hospital, durante el desarrollo de la experiencia se logró concientizar al personal hospitalario en este proceso, contando con su colaboración para la captura de la información debido a que es un proceso nuevo que demanda del uso de las historias clínicas y se estableció un trabajo mejor coordinado entre todo el personal que realiza su actividad alrededor de este documento.

Otra dificultad de orden técnico fue la adaptación de los equipos electrónicos para la captura de la información. Se resolvió la incompatibilidad de la página de internet con los exploradores en cada uno de los equipos de manera que es posible capturar toda la información. En el aparte de atención prehospitalaria se describen varias dificultades: no contar con un reporte escrito en la historia clínica de las actividades realizadas por los técnicos en urgencias médicas (TUM) en las ambulancias o en la escena, por esta razón debe encuestarse el TUM a su ingreso al servicio de urgencias para alimentar el módulo de atención prehospitalaria. En la plataforma se experimentaron inconvenientes para el registro de información en esta área por la implantación de cambios en el software, realizados en simultánea con la recolección con el fin de adaptar la base de datos a las necesidades de captura de información locales.

Es de destacar que los requerimientos de tiempo y de obtención de los datos, son diferentes cuando el documento fuente es la historia clínica en papel como es el caso del Hospital Público, en la que su disponibilidad, la legibilidad del documento y la interpretación de las notas implican más tiempo en la captura, comparado con el Hospital Privado, donde la historia en formato electrónico facilita y agiliza este proceso.

En la fase piloto de la implementación de un sistema de registro de trauma en Cali se identificaron las fortalezas y debilidades para alcanzar un sistema de información óptimo y útil. Se determinó las necesidades para la implementación del Sistema de Registro Trauma y los mecanismos para darle continuidad.

El registro se convierte en una fuente importante de información para el desarrollo de la investigación académica y productividad intelectual de los estudiantes y profesores de la Universidad del Valle.

En cada hospital logró implementarse el SRT e identificar las causas de consulta, morbilidad y muerte por trauma. El trauma secundario a caídas fue la principal causa de consulta en estos hospitales de referencia, representando un tercio de las consultas totales.

Disponer de las tendencias epidemiológicas en el manejo del trauma en cada centro permitirá una mejor planeación de los servicios de urgencias y del sistema regional de trauma con el fin de optimizar y de reducir los costos de atención en la ciudad. Permite proyectar la necesidad de un sistema de atención de trauma y emergencias en el área del Pacífico. 
Objective: To describes the experience in the implementation of a TRS in two hospitals in Cali, Colombia. Methods: The TRS includes prehospitalary, during hospitalization and discharging status information of each patient. Each hospital has an electronic data capture strategy. A three month Pilot-period descriptive analysis is presented. Results: 3293 patients has been registered, 1626 (49.4\%) from the Public hospital and 1613 (50.6\%) from the Private one. 67.2\% were men; the mean age \pm SD was 30.5 \pm 20 years; $30.5 \%$ were less than 18 years. The overall mortality rate was 3.5\%. The most frequent consulting cause were falls (33.7\%); $11.6 \%$ of injuries are secondary to fire gunshot, and this group where mortality rate was $62 \%$. Conclusion: It was determined the needing for the TRS implementation and the mechanisms to provide continuity. The registry becomes an information source for the investigation developing. It was identified the causes of consult, morbidity and death due to trauma that will allow a better planning of the emergency services and of the regional trauma system in order to optimize and reduce the attention costs. Based on optimal information system it will be able to present the necessary adjusts to redesign the Trauma and Emergencies Attention System in the Colombian South-West.

Key words: Wounds and injuries. Emergencies. Database. Registries. Eletronic health records.

\section{REFERENCIAS}

1. Aboutanos MB, Mora F, Rodas E, Salamea J, Parra MO, Salgado $\mathrm{E}$, et al. Ratification of IATSICNHO's guidelines for essential trauma care assessment in the South American region. World J Surg. 2010;34(11):2735-44

2. Departamento Administrativo de Planeación Municipal. Plan de Desarrollo Municipal 20122015 de Santiago - CaliDA: una ciudad para todos [online]. Santiago de Cali, Colômbia; 2012. [citado 29 mai 2012] Disponível em: http://www.cali.gov.co/ publicaciones. . php?id=44418

3. US Departament of Health and Human. The Data Bank. About Us [online]. Virginia, National Practitioner Data Bank [citado 29 mai. 2012] Disponível em: http://www.npdb-hipdb.hrsa.gov/ topNavigation/aboutUs.jsp

4. National Trauma Data Bank. Data center [online]. Chicago, American College of Surgeons [citado 29 mai. 2012] Disponível em: https://www.ntdbdatacenter.com/

5. Mora FE, Aboutanos MB. Uso de la tecnología en la creación de un Sistema de Trauma en Ecuador. Revista e Salud [Internet]. 2006 [citado 2012 Mar 16]; 2(8). disponível em: http:// www.revistaesalud.com/index.php/revistaesalud/article/view/ 125

6. Sociedad Panamericana de Trauma. Registro Panamericano de Trauma [online]. [citado 16 mar. 2012] Disponível em: http://www.panamtrauma.org/Default.aspx? pageld= 1098346 \& eventld=386763\&EventViewMode $=$ EventDetails

7. Panamerican Trauma Society. Registro de Trauma. ITSD Trauma Registry. VCU International Trauma System Development Program [online]. Richmond, EUA. [citado 29 mai. 2012] Disponível em: http:/ /pubapps.vcu.edu/itsdp-tr-mo/LOGIN.aspx?ReturnUrl=/itsdp-trmo/Default.aspx

8. Ferrada R, Rodriguez A, Peitzman A, Puyana JC, Ivatury R. Trauma Sociedad Panamericana de Trauma. $2^{a}$ ed. Bogotá: Distribuna; 2009.

9. Ordóñez CA, Ferrada R, Buitrago R. Cuidado intensivo y trauma. $2^{\text {a }}$ ed. Bogotá: Distribuna; 2009.

10. Peitzman AB, Rhodes M, Shwab CW, Yealy DM, Fabian TC, editores. Manual del Trauma: Trauma y cirugía de cuidado intensivo. Bogotá: Distribuna; 2011.

11. Pollock DA. Trauma registries and public health surveillance of injuries [online]. Trabalho apresentado no The International Collaborative Efforts Scientific Meeting; 1995 [citado 29 mai. 2012]; Washington, DC. Disponível em: http://www.cdc.gov/nchs/data/ ice/ice95v1/c11.pdf
12. NTDS. History of the New Data Standard [Internet]. [cited 2012 May 29]; Available from: http://www.ntdsdictionary.org/theNTDS/ historyofNTDS.html

13. Marina-Martínez L, Sánchez-Casado M, Hortiguela-Martin V, Taberna-Izquierdo MA, Raigal-Caño A, Pedrosa-Guerrero A, et al. «RETRATO» (REgistro de TRAuma grave de la provincia de TOledo): visión general y mortalidad. Med Intensiva [online] 2010;34(6):379-87

14. Nwomeh B, Lowell W, Kable R, Haley K, Ameh EA. Historia y desarrollo del registro de trauma: experiencia de los países desarrollados a los países en desarrollo [online]. World J Emerg Surg. 2006;1:32.

15. Brown K, Segrue M, Caldwell E, D'Amours S, Jalaludin B. South Western Sydney Regional Trauma Registry: Trauma 10-year report 1995-2004. Sydney: Liverpool Hospital; 2006.

16. Gómez VL, Caballero-Palomares M. Trauma ocular: distribución de acuerdo con la clasificación estandarizada. Trauma. 2002;5(1):510.

17. Weyll M, Silveira RC, Fonseca Júnior NL. Trauma ocular aberto: características de casos atendidos no Complexo Hospitalar Padre Bento de Guarulhos. Arq Bras Oftalmol. 2005;68(4):505 10

18. Correa MA, González G., Herrera MH. Orozco A. Epidemiología del trauma pediátrico en Medellín, Colombia 1992-1996. Colombia Med. 2000;31(2):77-80.

19. Ordoñez CA, Pino LF, Badiel M, Sánchez Al, Loaiza J, Ballestas L, et al. Safety of performing a delayed anastomosis during damage control laparotomy in patients with destructive colon injuries. J Trauma. 2011;71(6):1512-7; discussion 1517-8.

20. Ordoñez CA, Badiel M, Sánchez Al, Granados M, García AF, Ospina $\mathrm{G}$, et al. Improving mortality predictions in trauma patients undergoing damage control strategies. Am Surg. 2011;77(6):778-82.

21. Centers for Desease Controland Prevention. Injury prevention and control: data and statistics (WISQARSÔ) [online]. Atlanta, EUA [citado 02 mai. 2012] Disponível em: http://www.cdc.gov/injury/ wisqars/index.html

22. Demetriades D, Martin M, Salim A, Rhee P, Brown C, Doucet J et al. Relationship between American College of Surgeons trauma center designation and mortality in patients with severe trauma (injury severity score $>15$ ). J Am Coll Surg. 2006;202(2):212-5.

Recebido em 25/02/2012

Aceito para publicação em 31/03/2012

Conflito de interesse: nenhum

Fonte de financiamento: nenhuma 


\section{Como citar este artigo:}

Ordóñez CA, Pino LF, Tejada JW, Badiel M, Loaiza JH, Mata LV, Aboutanos MB. Experiencia en dos hospitales de tercer nível de atención del suroccidente de Colombia en la aplicación del registro internacional de trauma de la Sociedad Panamericana de Trauma (ITR/SPT-ITSDP. Rev Col Bras Cir. [periódico na Internet] 2012; 39(4).

Disponível em URL: http://www.scielo.br/rcbc

\section{Endereço para correspondência:}

Carlos a. Ordóñez

E-mail: ordonezcarlosa@gmail.com

RECURSOS: Este programa cuenta con recursos de investigación de la Universidad del Valle, Universidad ICESI y la Unidad de Investigaciones Clínicas de la Fundación Valle del Lili en colaboración con el Programa Internacional de Desarrollo de Sistemas de Trauma ITR/SPT-ITSDP de la Virginia Commonwealth University (VCU).
Contribución y agradecimientos:

Pregunta clínica, concepto y diseño: Ordoñez C.; Gonzalez A.; Pino L.; Badiel M. Gutierrez M.

Colección, ensamblaje, calidad de los datos: Tejada J.; Loaiza H.

Adquisición de los datos: Tejada J.; Pino L.; Calderón L.; Uribe A.; Muñoz L.( Análisis estadísticos: Cepeda M.; Loaiza H.( Análisis e interpretación de los datos: Cepeda M, Badiel M, Loaiza H.( Elaboración del manuscrito: Ordoñez C, Badiel M, Tejada J, Pino L, Loaiza H.( Revisión crítica del manuscrito para contenido intelectual relevante: Ordoñez C, Gonzalez A.; Pino L.; Badiel M, Loaiza H.; Gutierrez M. Supervisión del estudio y soporte técnico y logístico: Ordoñez $C$.; Pino L.; Badiel M.; Loaiza H. Obtención de recursos: Ordoñez C.; Gonzalez A.;Badiel M.

\section{Agradecimientos especiales a:}

- Las directivas del Hospital Universitario del Valle (hospital público) y de la Fundación Valle del Lili (Privado) por apoyar el proyecto. Al personal médico y paramédico de los servicios de urgencias, hospitalizaciones, cuidado intensivo y estadística de ambas instituciones. A Sandra Quenorán, Leidy Pedrosa, Lina Timote, Claudia Valencia, Shirley Valencia, Stephanie Sabogal y Jorge Olave por la recolección de los datos. 